\title{
GCU
}

Glasgow Caledonian

University

University for the Common Good

\section{Distal upper limb kinematics during functional everyday tasks}

Stansfield, Ben; Rooney, Scott; Brown, Lisa; Kay, Matthew; Spoettl, Lisa; Shanmugam, Sivaramkumar

Published in:

Gait and Posture

DOI:

10.1016/j.gaitpost.2018.01.004

Publication date:

2018

Document Version

Author accepted manuscript

Link to publication in ResearchOnline

Citation for published version (Harvard):

Stansfield, B, Rooney, S, Brown, L, Kay, M, Spoettl, L \& Shanmugam, S 2018, 'Distal upper limb kinematics during functional everyday tasks', Gait and Posture, vol. 61, pp. 135-140.

https://doi.org/10.1016/j.gaitpost.2018.01.004

\section{General rights}

Copyright and moral rights for the publications made accessible in the public portal are retained by the authors and/or other copyright owners and it is a condition of accessing publications that users recognise and abide by the legal requirements associated with these rights.

Take down policy

If you believe that this document breaches copyright please view our takedown policy at https://edshare.gcu.ac.uk/id/eprint/5179 for details of how to contact us. 
Title page

Title: Distal upper limb kinematics during functional everyday tasks

Authors names and affiliations

Ben Stansfield, Scott Rooney, Lisa Brown, Matthew Kay, Lisa Spoettl, Shivaramkumar Shanmugam

All authors are affiliated to: School of Health and Life Sciences, Glasgow Caledonian University, Cowcaddens Road, Glasgow, G4 OBA, UK.

Corresponding author

Ben Stansfield

Glasgow Caledonian University, Cowcaddens Road, Glasgow, G4 OBA, UK.

Email: ben.stansfield@gcu.ac.uk

Acknowledgements

The authors would like to thank all participants in the study. This research did not receive any specific grant from funding agencies in the public, commercial, or not-for-profit sectors. 


\section{Highlights (3-5 max 85 characters each)}

Minimal marker set used to track distal upper limb kinematics.

Elbow, wrist, thumb and metacarpophalangeal joint angles characterised.

Five functional tasks involving loading performed by healthy adults.

Normative joint angles presented numerically and graphically for the activity cycle.

Recommendations of particular activities to use in standardised assessments. 


\section{Abstract}

Quantitative characterisation of upper limb motion allows the evaluation of the effect of pathology on functional task performance, potentially directing rehabilitation strategies. Movement patterns of the distal upper limb in healthy adults during functional tasks have not been extensively characterised. During five loaded functional tasks (drinking from a glass, pouring from a kettle, turning a handle, lifting a bag to a shelf, turning a key) the movement patterns were characterised using three-dimensional motion analysis with a minimal marker set in 16 healthy adults $(10 \mathrm{M}, 6 \mathrm{~F}, 27$ (IQR:25-43)years). Joint angles reported include flexion/extension at the elbow and wrist, forearm supination/pronation and digits 2-5 metacarpophalangeal (MCP) joint flexion/extension.

Additionally for the thumb the angle between the metacarpal of the thumb and the $2^{\text {nd }}$ digit (Thumb base), the thumb MCP (Thumb MCP) and interphalangeal (Thumb IP) joint angles are presented. Durations of activities performed at self-selected comfortable speeds (3.36 (IQR:3.07,3.66)s turning a key to 6.20 (IQR:5.44,6.38)s drinking from a glass) are reported. The maximum joint angles used (median of participants' maxima) were $141^{\circ}$ of elbow flexion, $116^{\circ}$ forearm supination, $36^{\circ}$ wrist extension, $56^{\circ}$ Thumb base, $14^{\circ}$ Thumb MCP flexion, $18^{\circ}$ Thumb IP flexion, $85^{\circ}$ MCP2-5 flexion. The tasks of drinking from a glass, lifting a bag to a shelf and turning a key appeared to have the least variation in performance, suggesting that these activities are better suited to be selected as standardized tasks for assessing the impact of pathology on movement than pouring from a kettle and turning a handle.

\section{Keywords (up to 5)}

Upper limb kinematics; Metacarpophalangeal joint kinematics; Everyday activities; Range of motion; thumb; functional tasks. 


\section{Introduction}

Three-dimensional motion analysis can be used to characterise movement patterns of the upper limb during the execution of everyday tasks ${ }^{1}$. This information provides normative reference patterns that can be used to compare with the performance of these activities by those with pathology ${ }^{2-5}$. Deviations from the patterns of movement seen in persons without pathology may be used to inform rehabilitation strategies.

In the upper limb three-dimensional motion analysis has been used extensively to characterise movement of the shoulder, elbow and wrist ${ }^{1,6,7}$. The movements of the thumb and other digits are less well characterised, pointing to a need for further investigation to understand normative movement patterns and therefore evaluate deviations due to pathology. It is possible that compensation in one joint of the upper limb may occur due to deficit in movement range in another joint in the kinematic chain, meaning that is it important to have a clear understanding of normal movement patterns throughout the limb.

Tracking of the movements of the digits can be accomplished using a number of different methods. Invasive scanning techniques have been used to monitor digit kinematics ${ }^{8,9}$, but these cannot be implemented as a routine procedure. Also electrogoniometers have been used across the metacarpophalangeal joints ${ }^{10}$, but the attachment of these presents a considerable burden to participants and may affect movement patterns. Alternatively optoelectronic motion analysis systems can be used where markers attached to the skin are used to track movements of the underlying bones. Due to the difficulty of tracking complex movements (marker occlusion), full digit motion analysis has been restricted to simple tasks, often of little everyday relevance ${ }^{11}$. Motion of the metacarpophalangeal joints can, however, be monitored with a limited marker set extending only to the proximal interphalangeal joint ${ }^{12,13}$. Such a marker set is suitable for use in a range of functional tasks allowing the collection of reference data for normative movements. 
In this work the motion of the distal upper-limb (including elbow, wrist, thumb and metacarpophalangeal (MCP) joints) was characterised to enhance understanding of movements (joint angles and timings) in a selection of standardised everyday tasks. The joint movements used by a group for healthy adults were characterised as well as the variation of these movement patterns across the sample. This information can be used to evaluate the performance of these activities by those with pathology.

\section{Methods}

Participants

Participants were recruited from staff and students at a UK higher education establishment. All volunteers gave written informed consent with procedures approved by the institutional ethical review body. Only healthy participants with no current impairment of the upper limbs or neurological condition or uncorrected visual impairments were recruited. Previous impairment of upper limb function was not considered. Within the context of this report we use the term 'upper limb' to refer to all elements of the upper limb from shoulder to fingers. Participants age, gender, height, arm (from acromium process to fingertip arm outstretched), elbow to fingertip (from proximal elbow to fingertip elbow 90 degrees) and hand (distal wrist crease to fingertip, hand supinated wrist neutral) lengths were recorded. Only the results from participants who were right hand dominant are reported.

Motion analysis

All activities were tracked with a camera (Panasonic SDR-H85) synchronised with a motion analysis system (13 camera Qualisys, $120 \mathrm{~Hz}$ ) (Qualisys AB, Goteborg, Sweden). A marker set was used to 
allow determination of the joint angles of the upper limb including the elbow (flexion/extension and pronation/supination), wrist (flexion/extension), thumb (metacarpal 1 with respect to metacarpal 2 (Thumb base), metacarpophalangeal joint (Thumb MCP) and interphalangeal joint (Thumb IP)), and the other metacarpophalangeal joints (MCP2-5). This marker set was based on those previously proposed using a minimal marker set ${ }^{14,15}$. All markers (Table 1 ) were put in place for an initial static trial with the participant sitting at a table with palms placed on the table. For all subsequent trials the medial and lateral epicondyle markers (MEPI, LEPI) and the superior acromion marker (ACRM) were removed and the upper arm cluster used to identify their locations based on the STATIC trial.

Joint axes systems were defined (Table 1) and subsequently joint angles calculated (Visual 3D Professional V4.00.14, C-Motion, Germantown, MD, USA). Joint angles for elbow, wrist and digits 2 to 5 were calculated using a sequence of rotations from the proximal to the distal coordinate system (flexion/extension, then ulnar/radial deviation and then supination/pronation). As the definition of the thumb is more difficult than the other digits a specific method of determining joint angles was used as detailed in Table 1. Note that only selected outcomes are presented within this report.

\section{Activities}

The function of the upper limb can be categorised into three main object related actions, i.e. reach, grasp, and manipulation of objects ${ }^{16}$. Currently therapy assessment tools or intervention methods typically integrate all three actions of reach, grasp and manipulation of objects into one whole action. Therefore the five tasks selected for this study (Table 2) incorporated a variety of basic activities of daily living (ADL) tasks where the three object related hand actions could be described and categorised. Most ADL activities are bimanual and involve the use of both upper limbs. In spite of this, unimanual tasks were selected because clinicians and therapist typically assess and treat upper limb dysfunction on the affected upper limb or hand only. 
Test set up

A table (height $72 \mathrm{~cm}$ ) was used with a black none-reflective cloth covering it. An armless chair with wheels with height self-selected by the participant to allow comfortable performance of activities was used. Set locations were marked on the cloth for placement of equipment during the activities (F $40 \mathrm{~cm}, \mathrm{H} 60 \mathrm{~cm}$ straight in front and $\mathrm{E} 40 \mathrm{~cm}$ in front and $16 \mathrm{~cm}$ to the right) (See supplementary material Figure S1).

Test procedure

Each activity was repeated 3 times with the dominant arm. At the start and finish of each activity repetition the participants placed their hands, palm down on the table surface at a distance approximately $16 \mathrm{~cm}$ from the centre line. All activities were performed at a self-selected speed. Before each activity the participant was given oral description of the action required and then allowed to perform one or two practices of the action to ensure they understood the requirements. For the kettle pour and drinking from a glass the participants were instructed to mimic the actions (Table 2).

Segmentation of activities

Two time points were chosen for identification in each activity (Table 2). All time points were identified manually by observation of the movement of the motion markers within the motion analysis software (Qualisys AB, Goteborg, Sweden) and accompanying video images. 
Data analysis

Data were tested for normality of distribution (Shapiro-Wilk test, SPSS v23: SPSS Inc, Chicago, IL). Absolute and percentage timings of task components were determined based on the mean of each participant's data. The standardised starting point joint angles were characterised. For each activity $100 \%$ was defined as the time from initiation to completion. The median and interquartile ranges of joint angles from 0 to $100 \%$ of the activity were calculated based on all repetitions of all participants. Joint angles are presented graphically and maxima and minima are characterised with range. 


\section{Results}

For consistency of data presentation median and interquartile range (IQR) are used for all outcomes as there was a mix of normal and non-normal distributions within the data. Sixteen right hand dominant participants were recruited (10M, 6F), age 27 (IQR:25,43) years, height 176.0

(IQR:169.5,183.0)cm, arm length 77.0 (IQR:74.0-78.9)cm, elbow to fingertip 46.1 (IQR:44.9,49.6)cm and hand length 19.3 (IQR:17.7,20.0)cm.

All participants successfully completed each activity 3 times giving 48 repetitions of each activity. The activities took between 3.36 (IQR:3.07,3.66)s (turn key in lock) and 6.20 (IQR:5.44,6.38)s (drinking from a glass) to complete (Table 3). In general the activity lift bag to shelf was performed with a lower variation in time than the other activities, although to time point 2 in each activity the maximum interquartile range of timing was $0.55 \mathrm{~s}$ for pouring from a kettle. Time point 2 was achieved on average between 43.5 (IQR:39.7,46.6)\% (key turn) and 57.8 (IQR:55.7,62.1)\% (lift bag to shelf) of the total time.

Graphical representation of joint angles demonstrates the movement patterns required (Figure 1). The maxima and minima of joint angles are given in Table 4. As can be seen in Figure 1, the maxima and minima for numerous joint angles occurred across a range of time points. This was due to the participants maintaining one joint at a particular angle whilst making adjustments at other joints. Participants used the highest level of elbow flexion for drinking from a glass (141 (IQR:138,143) $\left.{ }^{\circ}\right)$, with very consistent maximum flexion $\left(106-108^{\circ}\right)$ for all other activities, although with varying minimum levels of flexion. Forearm supination in the key turn activity reached $116($ IQR:103,134) with a range of $96(\text { IQR:87,101 })^{\circ}$. Wrist flexion was slightly higher in the lift a bag to a shelf activity than the other activities, although this had considerable variability $\left(36(\operatorname{IQR}: 21,48)^{\circ}\right)$. The maximum flexion of the digits was reached in the pouring from a kettle activity and minimum levels in lifting the bag to the shelf. However, the Thumb base was maximum for the bag lift. 
Visual inspection of the median joint angle curves (Figure 1) illustrated high levels of variation for certain joint angles for certain activities. For example wrist flexion in lifting bag to shelf, Thumb MCP and Thumb IP joints for pouring from a kettle and Thumb IP for turning a handle. Graphically it appeared that MCP2-5 were often acting in synchrony across the activity cycle, although again high levels of variability are evident, especially for example in MCP5 for turning a handle and lifting a bag, this is reflected in high interquartile range values (Table 4).

\section{Discussion}

Characterisation of movement patterns during standardised activities provides reference data against which pathological movements can be compared. Despite challenges in performing motion analysis of the digits, five everyday activities were successfully characterised in sixteen participants with joint angles of the thumb and MCP joints included. Variation in performance of some activities was noted across the group, suggesting that a more strict performance protocol may be necessary to allow standardisation of performance.

The participants were asked to perform the activities at their self-selected speeds. The segmented and total times are therefore reflective of normal performance of these tasks. Some tasks were entirely specified in respect to the time course of performance. For example lifting a bag to a shelf involved reaching, grasping, lifting, placing and returning to the start position. Performance of this task may be considered to be tightly prescribed. In contrast, both drinking from a glass and pouring from a kettle included the instruction to mimic actions (drink/pour). This may have added to the variability in total time for these tasks. This might have been overcome with a more prescriptive instruction as to how to perform the activity, although this may have interfered with the natural performance of the task. Additionally some of the tasks had prescribed digit 2-5 grip configuration. For example, the kettle had an approximately cylindrical grip of $8.5 \mathrm{~cm}$ circumference. Therefore the 
digits 2-5 would have been constrained to this shape which may have determined the MCP joint angles. Also depending on grip type adopted the bag to shelf task may have constrained the MCP joint configuration. However, for all these tasks it would be possible to modify MCP joint configuration by adopting a different thumb opposition strategy in the grip. Therefore, the results as presented would provide a normative reference against which any deviations due to pathology could be established.

In comparison to previous studies the task of drinking from a glass has been reported with similar timing to that of the current study ${ }^{4,5,17}$. Previous studies reported peak elbow flexion from 115$136^{01,3,4,6,7,17}$, slightly less flexed than in the current study $\left(141(\operatorname{IQR} 138,143)^{\circ}\right)$. Reports of elbow pronation/supination appear to be more dependent on the chosen starting position of the arm and so it is difficult to directly compare with previous reports. There are varying reports of wrist joint angles during the drinking from a glass task: Gates et $\mathrm{al}^{18}$ and Beaudette and Chester ${ }^{6}$, present similar maximum extension to the current study $\left(30^{\circ}\right)$, however, other studies ${ }^{5,7,18,19}$ present smaller maximum wrist extension angles (below $20^{\circ}$ ). These differences perhaps reflect the use of different placement protocols for the glass, or potentially restrictions placed on movement by study protocols (including motion tracking system used). In previous reports of joint kinematics numerous other tasks have been used. Some of these have been similar to those presented here (e.g. 'Box off shelf ${ }^{18}$, 'turning $\mathrm{key}^{\prime 10}$, 'pouring water into a glass ${ }^{\prime 7}$ ). It is difficult to directly compare outcomes from this study to these other activities due to dissimilarities in the exact task movements. Hayashi and Shimizu ${ }^{10}$ report MCP joint maximum flexion between $66-78^{\circ}$ for digits $2-5$ for key turning, similar to the current study $\left(53-76^{\circ}\right)$. Similarly, for a range of activities of daily living including a key turning activity, Hume et al report MCP joint flexion of approximately $60^{\circ}$ with standard deviation of $8-12^{\circ}$ similar to the current study. Hume et $\mathrm{al}^{20}$ also report angles for the thumb, with thumb MCP and IP angles reported slightly higher than the current study (e.g. key activity MCP $20^{\circ}$, IP $16^{\circ}$ ) with a higher level of variability (SD approximately $15^{\circ}$ ). Lin et $\mathrm{al}^{21}$ reported higher thumb MCP and IP flexion angles to the current study for lateral pinch and power grip activities. These differences may 
be due to the configuration of test equipment or to the use of other measurement systems (e.g. electrogoniometers ${ }^{20}$, segment tripods ${ }^{21}$.

Where loading was applied to the hand during activities this was in a way that simulated routine activities, e.g. pouring from a kettle, lifting a bag or turning a door handle. It is likely therefore that the postures adopted by the participants reflect normal use which it might be assumed optimises joint orientation to efficiently generate force and minimise injury risk. Any alteration from these patterns may reflect the presence of pain, or musculoskeletal impairment, thus these data provide a useful reference against which the movements of those with pathology can be compared.

Higher joint angle variation at the Thumb base, Thumb MCP and Thumb IP joint was seen for the tasks of pouring from a kettle and turning a handle. Visual observation of the tasks confirmed that participants placed their thumbs in a variety of postures, including in direct opposition to the other digits, but also along the line of the kettle handle. Digit 5 appears to have higher variation in joint angle than the other digits, reflecting differences between participants in the choice of configuration of the hand for a number of the tasks. These natural variations in joint configurations in the hand for these particular tasks may make it more challenging to isolate modification of joint angles due to pathology.

Only the MCP joints of digits 2-5 were assessed. It was not possible to track the distal phalanges during several of the activities due to marker occlusion caused either by interference from the equipment being used or between the digits. Whilst three-dimensional joint angles at the joints described could have been derived from the marker set used ${ }^{15}$, only the primary joint angles have been presented. We have not made an attempt to examine the reliability within participants. This should be evaluated in future work to explore the repeatability of performance of these tasks both within and between sessions. It is possible that participant age, gender, strength and size may have affected outcomes. Results are presented here for the whole sample as one. Further exploration of 
the dependency of movement patterns on these variables would help our understanding of healthy adults' patterns of movement.

\section{Conclusion}

Distal upper limb joint angles were characterised during the performance of five functional tasks.

This was possible using a motion analysis protocol which included a minimal set of skin mounted markers. MCP and thumb joint angle characterisation was included to extend understanding of the use of these joints during everyday tasks. The tasks performed demonstrated a range of joint angles and variation in these angles across the sample. The tasks of drinking from a glass, lifting a bag to a shelf and turning a key appeared to have the least variation in performance, suggesting that these may be better standardised activities for assessing pathology than pouring from a kettle and turning a handle. 


\section{Conflict of interest}

The authors declare no conflict of interest.

\section{References}

1. Van Andel CJ, Wolterbeek N, Doorenbosch CAM, Veeger DHEJ, Harlaar J. Complete 3D kinematics of upper extremity functional tasks. Gait \& Posture 2008; 27: 120-127.

2. Jaspers E, Desloovere K, Bruyninckx H, Molenaers G, Klingels K, Feys H. Review of quantitative measurements of upper limb movements in hemiplegic cerebral palsy. Gait \& posture 2009; 30: 395404.

3. Kim K, Won-Kyung S, Lee J, Lee H-W, Sung Park D, Ko B-W, Kim J. Kinematic analysis of upper extremity movement during drinking in hemiplegic subjects. Clinical Biomechanics 2014; 29: 248256.

4. Murphy MA, Willen C, Sunnerhagen KS. Kinematic variables quantifying upper-extremity performance after stroke during reaching and drinking from a glass. Neurorehabilitation and Neural Repair 2011; 25(1): 71-80.

5. Reyes-Guzman A, Gli-Agudo A, Penasco-Martin B, Solis-Mazos M, Ama-Espinosa A, Perez-Rizo E. Kinematic analysis of the daily activity of drinking from a glass in a population with cervical spinal cord injury. Journal of NeuroEngineering and Rehabilitation 2010; 7: 41.

6. Beaudette B, Chester VL. Upper extremity kinematics in pediatric and young adult populations during activities of daily living. J Med \& Bio Eng 2014; 345): 448-454. 
7. Aizawa J, Masuda T, Koyama T, Nakamuru K, Isozaki K, Okawa A, Morita S. Three-dimensional motion of the upper extremity joints during various activities of daily living. J Biomech 2010; 43: 2915-2922.

8. Halilaj E, Rainbow MJ, Got C, Schwartz JB, Moore DC, Weiss A-PC, Ladd AL, Crisco JJ. In vivo kinematics of the thumb carpometacarpal joint during three isometric functional tasks. Clinical Orthopaedics and Related Research 2014; 472: 1114-1122.

9. Kuo L-C, Su F-C, Chiu H-Y, Yu C-Y. Feasibility of using a video-based motion analysis system for measuring thumb kinematics. Journal of Biomechanics 2002; 35: 1499-1506.

10. Hayashi $H$, Shimizu $H$. Essential motion of metacarpophalangeal joints during activities of daily living. Journal of Hand Therapy 2013; 26: 69-74.

11. Goislard de Mondabert B, Visser JMA, Vigouroux L. Comparison of three local frame definitions for the kinematic analysis of the fingers and the wrist. Journal of Biomechanics 2014; 47: 2590-2597.

12. Chappell PH, Metcalf CD, Burridge JH, Yule VT, Pickering RM. Constant angular velocity of the wrist during the lifting of a sphere. Journal of Medical Engineering \& Technology 2010; 34(4): 274284.

13. Wu G, van der Helm FCT, Veeger HEJ, Makhsous M, Van Roy P, Anglin C, Nagels J, Karduna AR, McQuade K, Wang X, Werner FW, Buchholz B. ISB recommendations on definitions of joint coordinate systems of various joints for the reporting of human joint motion - Part II: shoulder, elbow, wrist and hand. Journal of Biomechanics 2005; 38: 981-992.

14. Metcalf CD, Notley SV, Burridge JH, Chappell PH, Yule VT. Validation and application of a computational model for wrist and hand movements using surface markers. IEEE Transactions on Biomedical Engineering 2008; 55: 1199-1210. 
15. Carpinella I, Mazzoleni P, Rabuffetti M, Thorsen R, Ferrarin M. Experimental protocol for the kinematic analysis of the hand: definition and repeatability. Gait \& Posture 2006; 23(4): 445-454.

16. Kimmerle $M$, Mainwaring $L$, Borenstein $M$. The functional repertoire of the hand and its application to assessment. American Journal of Occupational Therapy. 2003; 57(5):489-98.

17. Murphy MA, Sunerhagen KS, Johnels B, Willen C. Three-dimensional kinematic motion analysis of a daily activity drinking from a glass: a pilot study. Journal of NeuroEngineering and Rehabilitation 2006; 3: 18.

18. Gates DH, Smurr Walters L, Cowley J, Wilken JM, Resnik L. Range of motion requirements for upper-limb activities of daily living. The American Journal of Occupational Therapy 2016;70(1):7001350010.

19. Aizawa J, Masuda T, Hyodo K, Jinno T, Yagishita K, Nakamuru K, Koyama T, Morita S. ranges of active joint motion for shoulder, elbow, and wrist in healthy adults. Disability \& Rehabilitation 2013; 35(16): 1342-1349.

20. Hume MC, Gellman H, McKellop H, Brumfield RH. Functional range of motion of the joints of the hand. Journal Hand Surgery Am 1990; 15(2): 240-243.

21. Lin HT, Kuo LC, Liu HY, Wu WL, Su FC, The three-dimensional analysis of three thumb joints coordination in activities of daily living. Clinical Biomechanics 2011;26(4):371-6. 
Table 1 Marker locations and definitions of segment coordinate systems and thumb angles

\begin{tabular}{ll}
\hline $\begin{array}{l}\text { Marker locations (markers on hand and forearm placed on dorsal aspect when hand resting palm down on table) } \\
\text { Marker }\end{array}$ & $\begin{array}{l}\text { Description of placement } \\
\text { WRU }\end{array}$ \\
WRR & Distal head of the ulnar \\
FAU & Dorsal aspect of the ulnar approximately $4 \mathrm{~cm}$ proximal to WRU \\
FAR & Dorsal aspect of the radius approx.. $4 \mathrm{~cm}$ proximal to WRR \\
CMC1, 2,5 & Proximal head of the $1^{\text {st }}, 2^{\text {nd }}$ and $5^{\text {th }}$ metacarpal at the carpometacarpal joint \\
CMC3,4* & Equally spaced between $\mathrm{CMC2}$ and 5 \\
MCP1 & Distal head of the $1^{\text {st }}-5^{\text {th }}$ metacarpals \\
IP & Distal head of the proximal phalanx of the thumb \\
FT1 & Distal head of the distal phalanx of the thumb \\
PIP2-5 & Distal head of the proximal phalanx of fingers $2-5$. \\
MEPI & Medial epicondyle of elbow (static trial only) \\
LEPI & Lateral epicondyle of elbow (static trial only) \\
ACRM & Superior acromion (static trial only) \\
UARM1-4 & Rigid upper arm cluster \\
\hline
\end{tabular}

*Calculated locations. All hand markers were $4 \mathrm{~mm}$ hemispheres, all other makers were $9 \mathrm{~mm}$ spheres.

\section{Segment coordinate system definitions}

\begin{tabular}{|c|c|c|c|}
\hline Segment & Origin & Axes & Tracking markers \\
\hline Upperarm & ACRM & $\begin{array}{l}Z=\text { from midEPI (mid-epicondyle markers) to ACRM pointing } \\
\text { proximally }\end{array}$ & $\begin{array}{l}\text { UARM1, UARM2, UARM3, } \\
\text { UARM4 }\end{array}$ \\
\hline
\end{tabular}

$\mathrm{Y}=$ perpendicular to both $\mathrm{Z}$ and line from midEPI to LEPI

pointing to back of person

$\mathrm{X}=$ perpendicular to both $\mathrm{Z}$ and $\mathrm{Y}$ pointing medially for right hand and laterally for left hand

\begin{tabular}{|c|c|c|c|}
\hline Forearm & mid EPI & $\begin{array}{l}Z \text { = from midW (mid-point of WRU and WRR) to midEPI pointing } \\
\text { proximally } \\
Y=\text { perpendicular to both } Z \text { and line from midW to WRU } \\
\text { pointing palmarly } \\
X=\text { perpendicular to both } Z \text { and } Y \text { pointing medially for right } \\
\text { hand and laterally for left hand when palm down on table }\end{array}$ & $\begin{array}{l}\text { midEPI, FAR, FAU, WRR, } \\
\text { WRU }\end{array}$ \\
\hline $\begin{array}{l}\text { MET1-5 } \\
\text { (Metacarpal } \\
\text { axes } \\
\text { systems) }\end{array}$ & relevant CMC & $\begin{array}{l}\mathrm{Z}=\text { from } \mathrm{MCP} \text { to } \mathrm{CMC} \text { pointing proximally } \\
\mathrm{Y}=\text { perpendicular to both } \mathrm{Z} \text { and line from } \mathrm{MET} 1: \mathrm{MCP} 1 \text { to } \mathrm{MCP} 2 \text {, } \\
\mathrm{MET} 2 \mathrm{MCP} 2 \text { to } \mathrm{MCP} 3, \mathrm{MET} 3: \mathrm{MCP} 2 \text { to } \mathrm{MCP} 3, \mathrm{MET} 4: \mathrm{MCP} 4 \text { to } \\
\mathrm{MCP5}, \mathrm{MET} 5: \mathrm{MCP} 4 \text { to } \mathrm{MCP} 5 \text { pointing palmarly } \\
\mathrm{X}=\text { perpendicular to both } \mathrm{Z} \text { and } \mathrm{Y} \text { pointing medially for right } \\
\text { hand when palm down on table }\end{array}$ & $\begin{array}{l}\text { MET1:CMC1, MCP1, } \\
\text { MCP2, MET2:CMC2, } \\
\text { MCP2, MCP3, } \\
\text { MET3:CMC3, MCP2, } \\
\text { MCP3, MET4:CMC4, } \\
\text { MCP4, MCP5, } \\
\text { MET5:CMC5, MCP4, } \\
\text { MCP5 }\end{array}$ \\
\hline $\begin{array}{l}\text { PPX2-5 } \\
\text { (Proximal } \\
\text { phalanx axis } \\
\text { systems) }\end{array}$ & relevant $\mathrm{MCP}$ & $\begin{array}{l}Z=P I P \text { to } M C P \text { pointing proximally } \\
Y=\text { perpendicular to both } Z \text { and line from } P P X 2: M C P 2 \text { to } M C P 3 \text {, } \\
\text { PPX3:MCP3 to MCP2, PPX4:MCP4 to MCP5, PPX5:MCP5 to } \\
\text { MCP4 pointing palmarly } \\
X=\text { perpendicular to both } Z \text { and } Y \text { pointing medially for right } \\
\text { hand when palm down on table }\end{array}$ & $\begin{array}{l}\text { PPX2:MCP2, MCP3, PIP2, } \\
\text { PPX3:MCP2, MCP3, PIP3, } \\
\text { PPX4:MCP4, MCP5, PIP4, } \\
\text { PPX5:MCP4, MCP5, PIP5 }\end{array}$ \\
\hline
\end{tabular}

Thumb angle definitions

$\begin{array}{ll}\text { Thumb base } & \text { Absolute angle between: CMC2-MCP2 CMC1-MCP1 } \\ \text { Thumb MCP (flexion/extension) } & \text { MCP1-IP in relation to line of CMC1-MCP1 } \\ \text { Thumb IP (flexion/extension) } & \text { IP-FT1 in relation to line of MCP1-IP }\end{array}$

The sense of the Thumb MCP and Thumb IP angles was determined by examining the thumb segment movements in relation to MCP5. 
Table 2 Activity descriptions with time points identified. (For locations E, F, H see supplementary material Figure S1)

\begin{tabular}{|c|c|c|c|c|c|}
\hline & \multirow[t]{2}{*}{ Activity } & \multirow[t]{2}{*}{ Location } & \multirow[t]{2}{*}{ Instructions } & \multicolumn{2}{|c|}{$\begin{array}{l}\text { Time points of } \\
\text { interest }\end{array}$} \\
\hline & & & & Point 1 & Point 2 \\
\hline 1 & $\begin{array}{l}\text { Drinking } \\
\text { from a glass }\end{array}$ & Glass starting point: $F$. & $\begin{array}{l}\text { Pick up glass, bring to be close to } \\
\text { lips, tip glass to simulate drinking, } \\
\text { return glass to starting point. } \\
150 \mathrm{ml} \text { water in glass (base } 5.7 \text {; top } \\
7.4 \text {; height } 8.8 \mathrm{~cm} \text { ). }\end{array}$ & $\begin{array}{l}\text { Initiation } \\
\text { of lift }\end{array}$ & 'Drinking' \\
\hline 2 & $\begin{array}{l}\text { Pouring } \\
\text { from a kettle } \\
\text { to a glass }\end{array}$ & $\begin{array}{l}\text { Start point of kettle: } E \\
\text { facing cup. Cup starting } \\
\text { point: } F .\end{array}$ & $\begin{array}{l}\text { Pick up kettle (weight } 1 \mathrm{~kg} \text { including } \\
\text { water, with sealed lid - water could } \\
\text { move within kettle, but not pour } \\
\text { out), perform action as if pouring } \\
\text { into a cup, return kettle to starting } \\
\text { point. }\end{array}$ & $\begin{array}{l}\text { Initiation } \\
\text { of lift }\end{array}$ & 'Pouring' \\
\hline 3 & $\begin{array}{l}\text { Turning a } \\
\text { door handle }\end{array}$ & $\begin{array}{l}\text { Lever style door handle } \\
(9.2 \mathrm{~cm} \text { long) at } \mathrm{H} \\
\text { ( } 20.5 \mathrm{~cm} \text { above table) } \\
\text { with resistance. }\end{array}$ & $\begin{array}{l}\text { Turn door handle to full extent } \\
\text { clockwise (from horizontal about } \\
80 \text { degrees of rotation). }\end{array}$ & $\begin{array}{l}\text { Turn } \\
\text { initiation }\end{array}$ & Turn end \\
\hline 4 & $\begin{array}{l}\text { Lifting a } 1 \mathrm{~kg} \\
\text { bag of sugar } \\
\text { onto a shelf }\end{array}$ & $\begin{array}{l}\text { Bag }(15.6 \times 9.8 \times 8.0 \mathrm{~cm}) \\
\text { starting point } E \text { to shelf } \\
\text { at } \mathrm{H}(40 \mathrm{~cm} \text { above table }) .\end{array}$ & Lift bag and place on shelf. & $\begin{array}{l}\text { Lift } \\
\text { initiation }\end{array}$ & $\begin{array}{l}\text { Bag on } \\
\text { shelf }\end{array}$ \\
\hline 5 & $\begin{array}{l}\text { Turning a } \\
\text { key in a lock }\end{array}$ & $\begin{array}{l}\text { Lock at } \mathrm{H}(20.5 \mathrm{~cm} \text { above } \\
\text { table). Yale type with } \\
\text { resistance. }\end{array}$ & $\begin{array}{l}\text { Key turned in clockwise direction } \\
\text { about } 45 \text { degrees to full extent. }\end{array}$ & $\begin{array}{l}\text { Turn } \\
\text { initiation }\end{array}$ & Turn end \\
\hline
\end{tabular}


Table 3 Total and time point timings of activities for all participants (median, interquartile range).

\begin{tabular}{|c|c|c|c|c|c|c|}
\hline \multirow{2}{*}{\multicolumn{2}{|c|}{ Activity }} & \multirow{2}{*}{$\begin{array}{l}\text { Total time } \\
\text { (s) }\end{array}$} & \multicolumn{2}{|c|}{ Time point 1} & \multicolumn{2}{|c|}{ Time point 2} \\
\hline & & & (s) & (\%) & (s) & (\%) \\
\hline \multirow[t]{2}{*}{1} & Drinking from a glass & & \multicolumn{2}{|c|}{ Lift initiation } & \multicolumn{2}{|c|}{ Point of drink } \\
\hline & & $6.20(5.44,6.38)$ & $1.19(1.10,1.37)$ & $20.9(18.9,21.9)$ & $2.67(2.51,2.93)$ & $45.4(42.6,46.8)$ \\
\hline \multirow[t]{2}{*}{2} & Pouring from kettle & & \multicolumn{2}{|c|}{ Lift initiation } & \multicolumn{2}{|c|}{ Point of pour } \\
\hline & & $5.89(5.40,6.34)$ & $1.16(1.02,1.35)$ & $19.9(18.0,20.9)$ & $2.78(2.55,3.10)$ & $49.2(45.0,50.7)$ \\
\hline \multirow[t]{2}{*}{3} & Turn handle & & \multicolumn{2}{|c|}{ Turn initiation } & \multicolumn{2}{|c|}{ Turn end } \\
\hline & & $3.60(3.35,4.10)$ & $1.15(1.02,1.31)$ & $31.0(29.5,33.1)$ & $1.63(1.47,1.77)$ & $43.1(40.7,47.8)$ \\
\hline \multirow[t]{2}{*}{4} & Lift bag to shelf & & \multicolumn{2}{|c|}{ Lift initiation } & \multicolumn{2}{|c|}{ Bag on shelf } \\
\hline & & $3.98(3.67,4.17)$ & $1.02(0.95,1.10)$ & $26.5(25.3,28.5)$ & $2.28(2.14,2.43)$ & $57.8(55.7,62.1)$ \\
\hline \multirow[t]{2}{*}{5} & Turn key in lock & & \multicolumn{2}{|c|}{ Turn initiation } & \multicolumn{2}{|c|}{ Turn end } \\
\hline & & $3.36(3.07,3.66)$ & $1.00(0.95,1.11)$ & $30.7(28.6,32.7)$ & $1.43(1.35,1.60)$ & $43.5(39.7,46.6)$ \\
\hline
\end{tabular}


Table 4 Joint angle characteristics (degrees) for all activities. Each outcome represents the value across $100 \%$ of the activity cycle. Values are calculated as the median (interquartile range) of all participants' outcomes. The start position is calculated as the median of all participants for all activities.

\begin{tabular}{|c|c|c|c|c|c|c|c|c|c|c|c|}
\hline \multirow{2}{*}{ Activity } & & \multicolumn{10}{|c|}{ Average angle (degrees) for all trials for each activity } \\
\hline & & 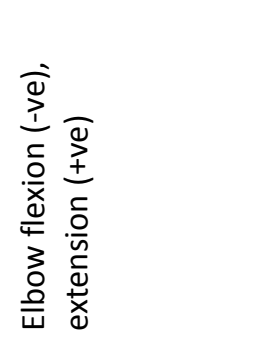 & 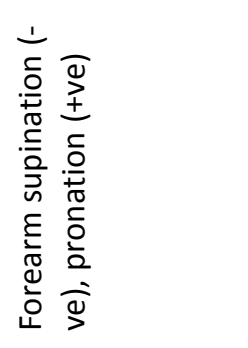 & 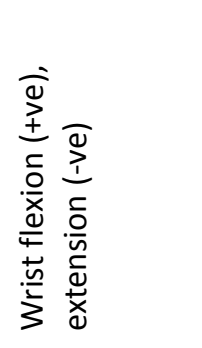 & 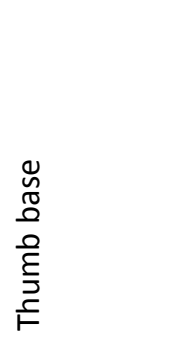 & 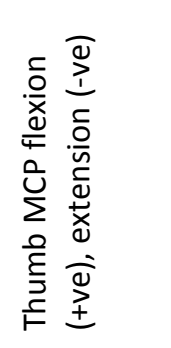 & 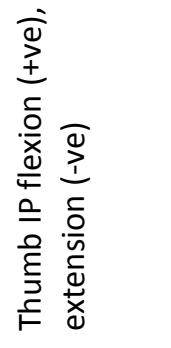 & 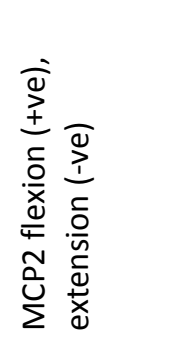 & 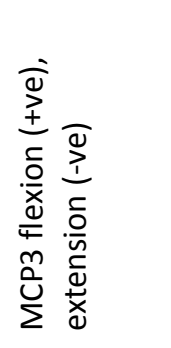 & 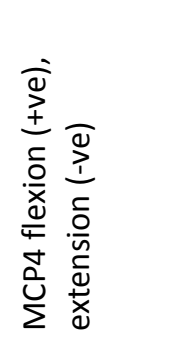 & 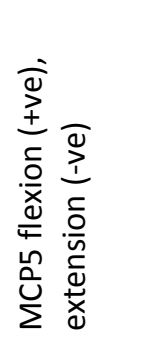 \\
\hline All & Start & $-101(-108,-94)$ & $-30(-36,-26)$ & $10(5,16)$ & $25(21,30)$ & $8(6,10)$ & $-3(-4,2)$ & $8(1,17)$ & $11(5,17)$ & $6(4,13)$ & $4(1,7)$ \\
\hline \multirow{3}{*}{$\begin{array}{l}1 \\
\text { Drink from } \\
\text { glass }\end{array}$} & Min & $-141(-143,-138)$ & $-101(-110,-89)$ & $-32(-34,-27)$ & $21(16,25)$ & $-4(-7,-1)$ & $-8(-11,-7)$ & $-2(-6,1)$ & $6(2,11)$ & $3(1,8)$ & $-2(-7,2)$ \\
\hline & Max & $-44(-50,-36)$ & $-27(-32,-21)$ & $14(8,21)$ & $52(47,58)$ & $11(8,13)$ & $11(8,14)$ & $32(23,39)$ & $56(47,59)$ & $54(48,58)$ & $57(40,63)$ \\
\hline & Range & $97(89,104)$ & $73(68,79)$ & $47(37,54)$ & $32(30,35)$ & $12(10,17)$ & $19(16,22)$ & $33(28,40)$ & $45(38,57)$ & $48(39,57)$ & $56(42,63)$ \\
\hline \multirow{3}{*}{$\begin{array}{l}2 \\
\text { Pour from } \\
\text { kettle }\end{array}$} & Min & $-107(-112,-103)$ & $-104(-109,-89)$ & $-32(-39,-28)$ & $23(18,26)$ & $4(-8,5)$ & $-6(-8,-4)$ & $1(-5,7)$ & $7(1,10)$ & $2(-1,6)$ & $-2(-7,1)$ \\
\hline & Max & $-43(-52,-32)$ & $-25(-31,-20)$ & $16(9,20)$ & $48(41,54)$ & $14(11,20)$ & $18(11,23)$ & $71(65,74)$ & $85(81,87)$ & $81(78,83)$ & $75(70,78)$ \\
\hline & Range & $65(50,75)$ & $75(59,81)$ & $49(44,53)$ & $27(20,29)$ & $14(9,21)$ & $24(20,27)$ & $69(63,77)$ & $79(71,84)$ & $78(72,82)$ & $78(74,82)$ \\
\hline \multirow{3}{*}{$\begin{array}{l}3 \\
\text { Turn } \\
\text { handle }\end{array}$} & Min & $-106(-113,-102)$ & $-88(-97,-78)$ & $-29(-35,-25)$ & $21(16,26)$ & $4(0,6)$ & $-9(-14,-5)$ & $3(-4,8)$ & $4(0,11)$ & $0(-2,6)$ & $-4(-9,0)$ \\
\hline & Max & $-37(-43,-32)$ & $-21(-29,-17)$ & $16(9,23)$ & $38(34,42)$ & $11(10,14)$ & $7(-3,20)$ & $64(55,77)$ & $83(71,92)$ & $78(62,86)$ & $64(45,79)$ \\
\hline & Range & $67(59,77)$ & $64(58,71)$ & $46(40,55)$ & $18(13,22)$ & $9(6,12)$ & $17(13,26)$ & $66(56,72)$ & $80(66,84)$ & $78(64,85)$ & $65(54,86)$ \\
\hline \multirow{3}{*}{$\begin{array}{l}4 \\
\text { Lift bag to } \\
\text { shelf }\end{array}$} & Min & $-107(-114,-103)$ & $-101(-118,-90)$ & $-36(-48,-21)$ & $21(16,27)$ & $-4(-7,-3)$ & $-8(-12,-6)$ & $-9(-12,-4)$ & $2(0,5)$ & $1(-2,4)$ & $-4(-7,0)$ \\
\hline & Max & $-31(-39,-23)$ & $-23(-30,-21)$ & $15(10,21)$ & $56(52,62)$ & $11(7,13)$ & $9(6,11)$ & $27(19,32)$ & $37(28,43)$ & $30(22,43)$ & $25(18,45)$ \\
\hline & Range & $74(67,86)$ & $75(66,88)$ & $54(36,66)$ & $36(32,39)$ & $13(10,20)$ & $17(14,21)$ & $35(27,42)$ & $31(26,42)$ & $29(23,39)$ & $29(21,42)$ \\
\hline \multirow{3}{*}{$\begin{array}{l}5 \\
\text { Turn key } \\
\text { in lock }\end{array}$} & Min & $-108(-114,-101)$ & $-116(-134,-103)$ & $-24(-29,-19)$ & $18(14,22)$ & $5(4,6)$ & $-7(-9,-5)$ & $5(-2,14)$ & $10(2,16)$ & $5(1,11)$ & $-1(-4,4)$ \\
\hline & Max & $-28(-37,-23)$ & $-21(-30,-16)$ & $12(7,25)$ & $31(26,34)$ & $12(11,14)$ & $11(7,13)$ & $53(48,55)$ & $73(70,78)$ & $76(73,79)$ & $76(68,79)$ \\
\hline & Range & $76(68,83)$ & $96(87,101)$ & $40(27,47)$ & $12(10,16)$ & $7(5,9)$ & $17(13,20)$ & $47(35,54)$ & $62(56,74)$ & $70(64,77)$ & $76(68,85)$ \\
\hline
\end{tabular}

*neutral wrist angles with palm facing backwards (down when resting on table top at start of activities) 


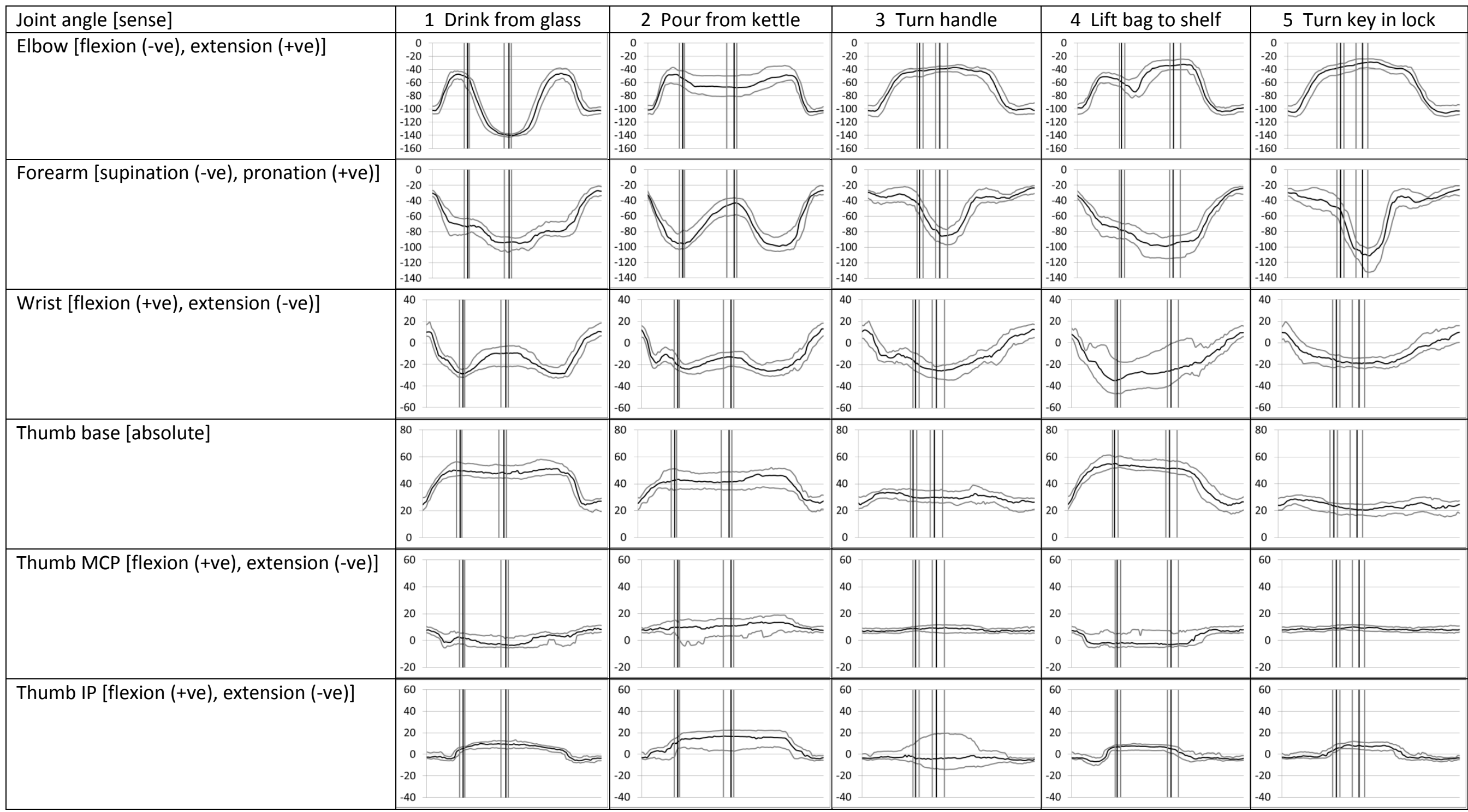

Figure 2 Joint angles (degrees) for all activities. Median and interquartile range are given across $100 \%$ of the activity time. Outcomes are calculated from all repeats of the activities across all the participants. Median and interquartile range of the timing points are illustrated by vertical lines (Table 3). 


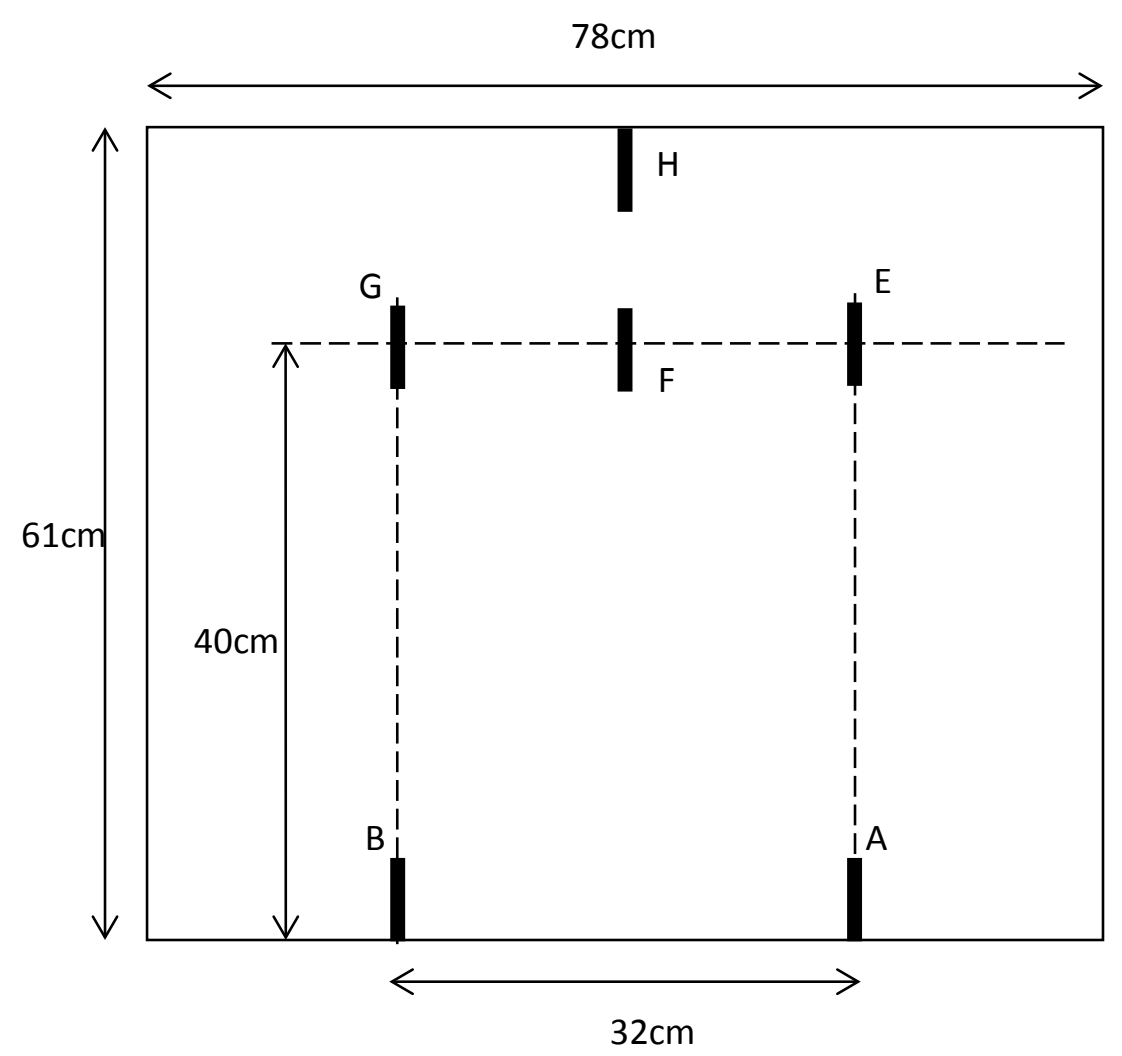

Figure S1 Location of task equipment. A table (height $72 \mathrm{~cm}$, depth $61 \mathrm{~cm}$, width $78 \mathrm{~cm}$ ) was used with a black none-reflective cloth covering it. The participant's location was at the bottom of the image. 